PROCEEDINGS OF THE

AMERICAN MATHEMATICAL SOCIETY

Volume 128, Number 2, Pages 599-604

$\mathrm{S}$ 0002-9939(99)05013-3

Article electronically published on July 7, 1999

\title{
TOWERS OF BOREL FUNCTIONS
}

\author{
JAMES HIRSCHORN
}

(Communicated by Alan Dow)

\begin{abstract}
We give mathematical reformulations of the cardinals $\mathfrak{p}$ and $\mathfrak{t}$ in terms of families of Borel functions. As an application we show that $\mathfrak{t}$ is invariant under the addition of a single Cohen real.
\end{abstract}

\section{INTRODUCTION}

Definitions and notation. Let $\mathbb{N}$ be the set of all nonnegative integers. We define a relation "almost set inclusion" on $\mathscr{P}(\mathbb{N})$ by

$$
A \subseteq^{*} B \text { iff }|A \backslash B|<\aleph_{0},
$$

where $A, B \subseteq \mathbb{N}$. And $A \supseteq^{*} B$ iff $B \subseteq{ }^{*} A$. For a family $\mathcal{F} \subseteq \mathscr{P}(\mathbb{N}), A \subseteq \mathbb{N}$ is a pseudo-intersection of $\mathcal{F}$ iff $A \subseteq^{*} F$ for every $F \in \mathcal{F}$.

A family of (infinite) subsets of $\mathbb{N}$ is called a filter base iff every nonempty finite subfamily has an infinite intersection. Let $\mathfrak{p}$ be the cardinality of the smallest filter base with no infinite pseudo-intersection. Let $\mathscr{P}_{\infty}(\mathbb{N})$ denote the set of all infinite subsets of $\mathbb{N}$. A tower is a subfamily of $\mathscr{P}_{\infty}(\mathbb{N})$ that is well-ordered by $\supseteq^{*}$. Let $\mathfrak{t}$ be the cardinality of the smallest tower with no infinite pseudo-intersection.

Now we define the corresponding notions in the realm of functions from $\mathbb{R}$ into $\mathscr{P}(\mathbb{N})$. For a property of the reals $P(x)$, we say that $P(x)$ for almost all $x$ in $\mathbb{R}$ iff there is a comeager $X \subseteq \mathbb{R}$ such that $P(x)$ holds for all $x \in X$. We define a relation on the set of all functions from $\mathbb{R}$ to $\mathscr{P}(\mathbb{N})$ by

$$
f \subseteq^{*} g \quad \text { iff } \quad f(x) \subseteq^{*} g(x) \text { for almost all } x \in \mathbb{R},
$$

where $f, g: \mathbb{R} \rightarrow \mathscr{P}(\mathbb{N})$. For a family $\mathcal{F}$ of functions from $\mathbb{R}$ to $\mathscr{P}(\mathbb{N}), f: \mathbb{R} \rightarrow$ $\mathscr{P}(\mathbb{N})$ is a pseudo-intersection of $\mathcal{F}$ iff $f \subseteq^{*} g$ for every $g \in \mathcal{F}$.

Definition 1.1. A family $\mathcal{F}$ of functions from $\mathbb{R}$ to $\mathscr{P}(\mathbb{N})$ is filtered iff for every nonempty finite subfamily $\mathcal{A} \subseteq \mathcal{F}$,

$$
\bigcap_{f \in \mathcal{A}} f(x) \text { is infinite for almost all } x \in \mathbb{R} .
$$

Definition 1.2. A family of functions $\mathcal{T}$ from $\mathbb{R}$ to $\mathscr{P}(\mathbb{N})$ is a tower iff

1. $\mathcal{T}$ is well-ordered by $\supseteq^{*}$, and

2. for every $f \in \mathcal{T}, f(x)$ is infinite for almost all $x \in \mathbb{R}$.

Received by the editors January 5, 1998 and, in revised form, March 30, 1998.

1991 Mathematics Subject Classification. Primary 03E10; Secondary 03E40, 28A20.

(C)1999 American Mathematical Society 
Denote the Cantor set, i.e. $\{0,1\}^{\mathbb{N}}$ with the product topology, by $\mathscr{C}$. We give $\mathscr{P}(\mathbb{N})$ a topology by identifying $\mathscr{P}(\mathbb{N})$ with $\mathscr{C}$.

Definition 1.3. Let $\mathfrak{p}_{1}$ be the cardinality of the smallest filtered family $\mathcal{F}$ of Borel functions from $\mathbb{R}$ to $\mathscr{P}(\mathbb{N})$ such that there is no Borel function $f: \mathbb{R} \rightarrow \mathscr{P}_{\infty}(\mathbb{N})$ which is a pseudo-intersection of $\mathcal{F}$.

Our first claim is that $\mathfrak{p}=\mathfrak{p}_{1}$.

Definition 1.4. Let $\mathfrak{t}_{1}$ be the cardinality of the smallest tower $\mathcal{T}$ of Borel functions from $\mathbb{R}$ to $\mathscr{P}(\mathbb{N})$ such that there is no Borel function $f: \mathbb{R} \rightarrow \mathscr{P}_{\infty}(\mathbb{N})$ which is a pseudo-intersection of $\mathcal{T}$.

The other main result of this paper is that $\mathfrak{t}=\mathfrak{t}_{1}$. No specialized knowledge of set theory is required in order to understand the proofs.

I wish to thank Stevo Todorčević for his handwritten notes: " $\mathscr{C}_{\omega} \|-\dot{\mathfrak{p}} \leq \check{\mathfrak{p}}$ " from the summer of 1997.

\section{THE PROOF}

To start, we need the following basic facts about $\mathfrak{p}$ and $\mathfrak{t}$. Let $\mathbb{N}^{\mathbb{N}}$ be the set of all functions from $\mathbb{N}$ to $\mathbb{N}$. We define the relation $\leq^{*}$ on $\mathbb{N}^{\mathbb{N}}$ by

$$
f \leq^{*} g \text { iff there is an } n \in \mathbb{N} \text { such that } f(k) \leq g(k) \text { for all } k \geq n,
$$

where $f, g \in \mathbb{N}^{\mathbb{N}}$. Let $\mathfrak{b}$ be the size of the smallest subfamily of $\mathbb{N}^{\mathbb{N}}$ that is unbounded in $\left\langle\mathbb{N}^{\mathbb{N}}, \leq^{*}\right\rangle$.

Theorem 2.1. $\omega_{1} \leq \mathfrak{p} \leq \mathfrak{t} \leq \mathfrak{b}$.

Proof. See [2].

We also use the following trivial fact.

Fact 2.2. $\mathfrak{t}$ is regular.

Proof. See [2].

Let $\mathcal{I}$ be an ideal. Define

$$
\operatorname{add}(\mathcal{I})=\min \{|\mathcal{A}|: \mathcal{A} \subseteq \mathcal{I} \text { and } \bigcup \mathcal{A} \notin \mathcal{I}\}
$$

For a topological space $X$, we let $\mathcal{M}$ denote the $\sigma$-ideal of meager subsets of $X$. We shall need the following lower bound for $\operatorname{add}(\mathcal{M})$.

Theorem 2.3 (Piotrowski-Szymański). For any Polish space, $\operatorname{add}(\mathcal{M}) \geq \mathfrak{t}$.

Proof. See [4].

Theorem 2.4. $\mathfrak{p}=\mathfrak{p}_{1}$.

Theorem 2.5. $\mathfrak{t}=\mathfrak{t}_{1}$.

We prove Theorems 2.4 and 2.5 simultaneously.

Proofs. $\mathfrak{t}_{1} \leq \mathfrak{t}$ : Let $\left\{A_{\xi}\right\}_{\xi<\mathfrak{t}} \subseteq \mathscr{P}_{\infty}(\mathbb{N})$ be a tower with no infinite pseudo-intersection, such that the enumeration respects the well-ordering of the tower, i.e. $\xi \leq \eta \rightarrow$ $A_{\eta} \subseteq^{*} A_{\xi}$. For each $\xi<\mathfrak{t}$, define $f_{\xi}: \mathbb{R} \rightarrow \mathscr{P}_{\infty}(\mathbb{N})$ by $f_{\xi}(x)=A_{\xi}$ for all $x \in \mathbb{R}$. Then obviously $\left\{f_{\xi}\right\}_{\xi<t}$ is a tower of Borel functions. We need to show that there is no Borel function $f: \mathbb{R} \rightarrow \mathscr{P}_{\infty}(\mathbb{N})$ which is a pseudo-intersection of $\left\{f_{\xi}\right\}_{\xi<t}$. 
Suppose to the contrary that $f$ is such a function. Find a comeager $G \subseteq \mathbb{R}$ such that $f\lceil G$ is continuous. For each $\xi<\mathfrak{t}$ and $n \in \mathbb{N}$, define

$$
F_{\xi n}=\left\{x \in G: f(x) \backslash n \subseteq A_{\xi}\right\} .
$$

Then each $F_{\xi n}$ is a relatively closed subset of $G$. Since $\bigcup_{n=0}^{\infty} F_{\xi n}$ is a relatively comeager - and in particular, nonmeager - subset of $G$, there is a nonempty rational interval $I_{\xi}$ and an $n_{\xi} \in \mathbb{N}$ such that

$$
G \cap I_{\xi} \subseteq F_{\xi n_{\xi}} .
$$

Since $\mathfrak{t}$ is an uncountable regular cardinal (Theorem 2.1 and Fact 2.2), there are $\bar{n} \in \mathbb{N}$ and a rational interval $\bar{I}$ such that

$$
\Gamma=\left\{\xi<\mathfrak{t}: n_{\xi}=\bar{n} \text { and } I_{\xi}=\bar{I}\right\}
$$

is cofinal in t. Pick $x \in G \cap \bar{I}$. Then $f(x) \backslash \bar{n} \subseteq \bigcap_{\xi \in \Gamma} A_{\xi}$, from which it follows that $f(x)$ is an infinite pseudo-intersection of $\left\{A_{\xi}\right\}_{\xi<t}$, a contradiction.

$\mathfrak{p}_{1} \leq \mathfrak{p}$ : First suppose that $\mathfrak{p}=\mathfrak{t}$. Note that every tower of Borel functions is also filtered. Hence $\mathfrak{p}_{1} \leq \mathfrak{t}_{1} \leq \mathfrak{t}=\mathfrak{p}$.

By Theorem 2.1, it remains to consider the case where $\mathfrak{p}<\mathfrak{t}$. Let $\left\{A_{\xi}\right\}_{\xi<\mathfrak{p}} \subseteq$ $\mathscr{P}(\mathbb{N})$ be a filter base with no infinite pseudo-intersection. For each $\xi<\mathfrak{p}$, define $f_{\xi}: \mathbb{R} \rightarrow \mathscr{P}(\mathbb{N})$ by $f_{\xi}(x)=A_{\xi}$ for all $x \in \mathbb{R}$. Obviously $\left\{f_{\xi}\right\}_{\xi<\mathfrak{p}}$ is a filtered family of Borel functions. Suppose towards a contradiction that $f: \mathbb{R} \rightarrow \mathscr{P}_{\infty}(\mathbb{N})$ is a pseudo-intersection of $\left\{f_{\xi}\right\}_{\xi<\mathfrak{p}}$. For each $\xi<\mathfrak{p}$, choose a comeager $G_{\xi} \subseteq \mathbb{R}$ such that $f(x) \subseteq^{*} A_{\xi}$ for all $x \in G_{\xi}$. By assumption and Theorem 2.3, $\mathfrak{p}<\operatorname{add}(\mathcal{M})$, whence there is a comeager $G \subseteq \bigcap_{\xi<p} G_{\xi}$. But if we take $x \in G$, then $f(x)$ is an infinite pseudo-intersection of $\left\{A_{\xi}\right\}_{\xi<\mathfrak{p}}$, giving a contradiction.

$\mathfrak{p} \leq \mathfrak{p}_{1}, \mathfrak{t} \leq \mathfrak{t}_{1}$ : We take $\kappa<\mathfrak{p}(\kappa<\mathfrak{t})$, and prove that $\kappa<\mathfrak{p}_{1}\left(\kappa<\mathfrak{t}_{1}\right)$. Note that $\mathbb{R}$ is homeomorphic to the unit interval $(0,1)$ via the standard homeomorphism. Consider the standard surjection $\Phi: \mathscr{C} \rightarrow[0,1]$, where $\Phi(x)$ is the base 2 expansion of $x$ after the decimal point, for all $x \in \mathscr{C}$. $\Phi$ is a homeomorphism on a co-countable subset of $\mathscr{C}$. Hence, if we replace the domains of the functions in the formulations of $\mathfrak{p}_{1}$ and $\mathfrak{t}_{1}$ with $\mathscr{C}$, then we are proving an equivalent result.

Suppose that $f_{\xi}: \mathscr{C} \rightarrow \mathscr{P}(\mathbb{N})(\xi<\kappa)$ is a filtered family (tower) of Borel functions. (For $\left\{f_{\xi}\right\}_{\xi<\kappa}$ a tower, we assume that the enumeration respects the well-ordering of the tower.) For each $\xi<\kappa$, we can find a dense $G_{\delta}$-and thus comeager - set $G_{\xi} \subseteq \mathscr{C}$ such that $f_{\xi} \uparrow G_{\xi}$ is continuous. For each nonempty $F \in$ $[\kappa]^{<\aleph_{0}}$, write $F=\left\{\xi_{1}<\xi_{2}<\cdots<\xi_{n}\right\}$. Then there is a comeager $G_{F} \subseteq \mathscr{C}$ such that $\left|\bigcap_{i=1}^{n} f_{\xi_{i}}(x)\right|=\aleph_{0}$ for all $x \in G_{F}$ (and for a tower, $f_{\xi_{j}}(x) \subseteq^{*} f_{\xi_{i}}(x)$ for all $1 \leq i<j \leq n$, for all $x \in G_{F}$ ).

By Theorem 2.3, there is a dense $G_{\delta}$ set $H \subseteq \mathscr{C}$ such that

$$
H \subseteq \bigcap_{\xi<\kappa} G_{\xi} \cap \bigcap_{F \in[\kappa]<\aleph_{0}} G_{F} .
$$

Then

$$
\text { for all } \xi<\kappa, f_{\xi} \uparrow H \text { is continuous, and }
$$$$
\text { for all } x \in H,\left\{f_{\xi}(x)\right\}_{\xi<\kappa} \text { is a filter base, }
$$$$
\text { for all } x \in H,\left\{f_{\xi}(x)\right\}_{\xi<\kappa} \text { is a tower. }
$$

Let $\left\{x_{n}\right\}_{n=0}^{\infty}$ be an enumeration of a dense subset of $H$. 
Since $\kappa<\mathfrak{p}\left(\kappa<\mathfrak{t}\right.$ ), by $(2)$ (by $\left(2^{\prime}\right)$ ), there is a sequence $\{d(n)\}_{n=0}^{\infty} \subseteq \mathscr{P}_{\infty}(\mathbb{N})$ such that

$$
d(n) \subseteq^{*} f_{\xi}\left(x_{n}\right) \quad \text { for all } n \in \mathbb{N} \text {, and all } \xi<\kappa .
$$

For the remainder of the proof, we need only the fact that $\kappa<\mathfrak{b}$. By (3), for each $\xi<\kappa$, we can choose $g_{\xi}: \mathbb{N} \rightarrow \mathbb{N}$ so that $d(n) \backslash g_{\xi}(n) \subseteq f_{\xi}\left(x_{n}\right)$ for all $n \in \mathbb{N}$. Since $\kappa<\mathfrak{b}$, there is a $D: \mathbb{N} \rightarrow \mathbb{N}$ such that $g_{\xi} \leq^{*} D$ for all $\xi<\kappa$. For each $\xi<\kappa$, fix $m_{\xi} \in \mathbb{N}$ so that $g_{\xi}(n) \leq D(n)$ for all $n \geq m_{\xi}$. Then

$$
d(n) \backslash D(n) \subseteq f_{\xi}\left(x_{n}\right) \text { for all } \xi<\kappa \text {, for all } n \geq m_{\xi} .
$$

Now we measure the continuity of $f_{\xi} \uparrow H$ at each $x_{n}$.

Notation. For $t \in 2^{<\mathbb{N}}$ and $A \subseteq \mathscr{C},[t]_{A}=[t] \cap A$.

Claim 5. There is a function $F: \mathbb{N} \times \mathbb{N} \rightarrow \mathbb{N}$ such that for all $\xi<\kappa$,

$$
f_{\xi}^{\prime \prime}\left[x _ { n } \lceil F ( n , \ell ) ] _ { H } \subseteq \left[f_{\xi}\left(x_{n}\right)\lceil\ell] \quad \text { for all but finitely many }(n, \ell) \in \mathbb{N} \times \mathbb{N},\right.\right.
$$

i.e. $\left[f_{\xi}\left(x_{n}\right)\lceil\ell]=\left\{A \subseteq \mathbb{N}: f_{\xi}\left(x_{n}\right) \cap \ell \sqsubseteq A\right\}\right.$, where $A \sqsubseteq B$ means that $A$ is an initial segment of $B$ for $A, B \subseteq \mathbb{N}$.

Proof. Let $\Phi: \mathbb{N} \rightarrow \mathbb{N} \times \mathbb{N}$ be a bijection. For each $\xi<\kappa$, since $f_{\xi}\lceil H$ is continuous, there is a function $F_{\xi}: \mathbb{N} \times \mathbb{N} \rightarrow \mathbb{N}$ such that

$$
f_{\xi}^{\prime \prime}\left[x_{n}\left\lceil F_{\xi}(n, \ell)\right]_{H} \subseteq\left[f_{\xi}\left(x_{n}\right) \uparrow \ell\right]\right.
$$

for all $n, \ell \in \mathbb{N}$. For each $\xi<\kappa$, define $g_{\xi}: \mathbb{N} \rightarrow \mathbb{N}$ by $g_{\xi}(m)=F_{\xi}(\Phi(m))$ for all $m \in \mathbb{N}$. Since $\kappa<\mathfrak{b}$, there is an $h: \mathbb{N} \rightarrow \mathbb{N}$ so that $g_{\xi} \leq^{*} h$ for all $\xi<\kappa$. Define $F: \mathbb{N} \times \mathbb{N} \rightarrow \mathbb{N}$ by $F(n, \ell)=h\left(\Phi^{-1}(n, \ell)\right)$ for all $n, \ell \in \mathbb{N}$.

Let $\left\{s_{i}\right\}_{i=0}^{\infty}$ enumerate $2^{<\mathbb{N}}$. We define $\left\{n_{i}\right\}_{i=0}^{\infty},\left\{k_{i}\right\}_{i=0}^{\infty} \subseteq \mathbb{N}$, and $\left\{t_{i}\right\}_{i=0}^{\infty} \subseteq 2^{<\mathbb{N}}$ by recursion on $i$ so that for all $i \in \mathbb{N}$,

(a) $s_{i} \subseteq x_{n_{i}}$,

(b) $n_{i} \geq i$,

(c) $k_{i} \in d\left(n_{i}\right)$

(d) $k_{i} \geq D\left(n_{i}\right)$,

(e) $k_{i+1}>k_{i}$,

(f) $t_{i} \subseteq x_{n_{i}}$, and

(g) $\left|t_{i}\right| \geq \max \left(F\left(n_{i}, k_{i}+1\right),\left|s_{i}\right|\right)$.

Let $i \in \mathbb{N}$ be given. Since $\left\{x_{n}\right\}_{n=0}^{\infty}$ is dense, there are infinitely many $x_{n}$ 's extending $s_{i}$. Hence we can find $n_{i} \geq i$ such that $s_{i} \subseteq x_{n_{i}}$. Let $k_{i} \in d\left(n_{i}\right)$ be sufficiently large so that (d) and (e) hold. Then obviously we can find $t_{i}$ as required.

Define $G^{*} \subseteq \mathscr{C}$ by

$$
G^{*}=\bigcap_{m=0}^{\infty} \bigcup_{i=m}^{\infty}\left[t_{i}\right]
$$

By (a), (f), and (g), $t_{i} \supseteq s_{i}$ for all $i \in \mathbb{N}$. It follows that $G^{*}$ is a dense $G_{\delta}$ set. Hence $G=G^{*} \cap H$ is also dense $G_{\delta}$. Now we define $f: G \rightarrow \mathscr{P}_{\infty}(\mathbb{N})$ by

$$
f(x)=\left\{k_{i}: i \in \mathbb{N}, t_{i} \subseteq x\right\} .
$$

By (e), $f(x)$ is infinite for all $x \in G$, whence $f$ is well-defined.

Claim 6. For every $x \in G, f(x) \subseteq^{*} f_{\xi}(x)$ for all $\xi<\kappa$. 
Proof. Take $x \in G$ and $\xi<\kappa$. By Claim 5, there is an $\ell_{\xi} \in \mathbb{N}$ such that

$$
f_{\xi}^{\prime \prime}\left[x _ { n } \lceil F ( n , \ell ) ] _ { H } \subseteq \left[f_{\xi}\left(x_{n}\right)\lceil\ell] \text { for all } n \in \mathbb{N} \text {, and all } \ell>\ell_{\xi} .\right.\right.
$$

We claim that

$$
f(x) \backslash\left(\ell_{\xi} \cup\left\{k_{i}: i<m_{\xi}\right\}\right) \subseteq f_{\xi}(x) .
$$

Suppose that $i \in \mathbb{N}$ is such that $k_{i} \in f(x) \backslash\left(\ell_{\xi} \cup\left\{k_{i}: i<m_{\xi}\right\}\right)$. By $(\mathrm{g}),\left|t_{i}\right| \geq$ $F\left(n_{i}, k_{i}+1\right)$. Therefore, since $t_{i} \subseteq x_{n_{i}}$ and $k_{i}+1>\ell_{\xi}$,

$$
f_{\xi}^{\prime \prime}\left[t_{i}\right]_{H}=f_{\xi}^{\prime \prime}\left[x_{n_{i}} \uparrow\left|t_{i}\right|\right]_{H} \subseteq\left[f_{\xi}\left(x_{n_{i}}\right)\left\lceil k_{i}+1\right] .\right.
$$

Since $i \geq m_{\xi}, n_{i} \geq m_{\xi}$ by (b). Hence by (4), (c), and (d), $k_{i} \in f_{\xi}\left(x_{n_{i}}\right)$. Thus $k_{i} \in A$ for all $A \in\left[f_{\xi}\left(x_{n_{i}}\right)\left\lceil k_{i}+1\right]\right.$. Since $t_{i} \subset x$ and $x \in H, x \in\left[t_{i}\right]_{H}$. Therefore $f_{\xi}(x) \in\left[f_{\xi}\left(x_{n_{i}}\right)\left\lceil k_{i}+1\right]\right.$, whence $k_{i} \in f_{\xi}(x)$.

Claim 7. $f$ is continuous.

Proof. Take $x \in G$. Given $\ell \in \mathbb{N}$, we need to find an $n \in \mathbb{N}$ such that $f^{\prime \prime}\left[x\lceil n]_{G} \subseteq\right.$ $[f(x) \mid \ell]$. By $(\mathrm{e}), n=\max \left\{\left|t_{i}\right|: k_{i}<\ell\right\}$ is finite. Fix $y \in\left[x\lceil n]_{G}\right.$. Clearly, for all $k<\ell, k \in f(x)$ iff $k \in f(y)$. Thus $f(y) \in[f(x)\lceil\ell]$.

Extend the domain of $f$ by defining $f(x)=\mathbb{N}$ for all $x \notin G$. Then by Claim 7 , and since $G$ is $G_{\delta}, f$ is Borel. And by Claim $6, f(x)$ is a pseudo-intersection of $\left\{f_{\xi}(x)\right\}_{\xi<\kappa}$ for all $x \in G$. Since $G$ is comeager, $f \subseteq^{*} f_{\xi}$ for all $\xi<\kappa$. Therefore $f$ is a pseudo-intersection of the family of functions $\left\{f_{\xi}\right\}_{\xi<\kappa}$. This completes the proof that $\kappa<\mathfrak{p}_{1}\left(\kappa<\mathfrak{t}_{1}\right)$.

\section{Adding a single Cohen Real}

Definitions and notation. The poset for adding a single Cohen real is viewed as the poset of finite partial functions from $\mathbb{N}$ into 2 which we denote by $\mathscr{C}_{\omega}$. If $f: \mathscr{C} \rightarrow \mathscr{P}(\mathbb{N})$ is a Borel function, then $\tilde{f}$ is a name for the decoding of $f$ in the forcing extension. Fix $\mathscr{C}_{\omega}$-names $\dot{\mathfrak{p}}$ and $\dot{\mathfrak{t}}$ which are forced to be the values of $\mathfrak{p}$ and $\mathfrak{t}$ in the extension by one Cohen real, respectively. Let $\dot{c}$ be the canonical name for the Cohen real.

There is a canonical correspondence between names for reals in the Cohen extension and codes for Borel functions from $\mathscr{C}$ into $\mathscr{P}(\mathbb{N})$. We describe this by $\dot{x} \mapsto f_{\dot{x}}$, where $\mathscr{C}_{\omega} \| \tilde{f}_{\dot{x}}(\dot{c})=\dot{x}$ (see [3]). And in the other direction we have: $f \mapsto \dot{x}_{f}$, where $\mathscr{C}_{\omega} \|-\tilde{f}(\dot{c})=\dot{x}_{f} \subseteq \check{\mathbb{N}}$. Moreover, it is an easy exercise to verify that given any two names $\dot{x}$ and $\dot{y}$ for reals,

$$
\mathscr{C}_{\omega} \|-\dot{x} \subseteq^{*} \dot{y} \quad \text { iff } \quad f_{\dot{x}} \subseteq^{*} f_{\dot{y}} .
$$

Also, for any finite sequence $\dot{x}_{1}, \ldots, \dot{x}_{n}$ of names for reals,

$$
\mathscr{C}_{\omega} \Vdash\left|\bigcap_{k=1}^{n} \dot{x}_{k}\right|=\aleph_{0} \quad \text { iff } \quad\left|\bigcap_{i=1}^{n} f_{\dot{x}_{k}}(y)\right|=\aleph_{0} \text { for almost all } y .
$$

For example, if $\left\{\dot{x}_{\xi}\right\}_{\xi<\kappa}$ is a name for a tower, then by (3.1) and (3.2), $\left\{f_{\dot{x}_{\xi}}\right\}_{\xi<\kappa}$ is a tower of Borel functions. In this manner it is easily checked that

(3.3) $\mathscr{C}_{\omega} \Vdash \dot{\mathfrak{p}}=\check{\mathfrak{p}}_{1}$, and

(3.4) $\mathscr{C}_{\omega} \Vdash \dot{\mathfrak{t}}=\check{\mathfrak{t}}_{1}$.

In effect, we are viewing $\mathscr{C}_{\omega}$-names as Borel codes (e.g. the code for $f_{\dot{x}}$ ), with the above translation yielding $\dot{\mathfrak{p}}=\check{\mathfrak{p}}_{1}$ and $\dot{\mathfrak{t}}=\check{\mathfrak{t}}_{1}$. By (3.3) and (3.4), the following Corollaries are immediate from Theorems 2.4 and 2.5 . 
Corollary 3.5. $\mathscr{C}_{\omega} \Vdash \dot{\mathfrak{p}}=\check{\mathfrak{p}}$.

Corollary 3.6. $\mathscr{C}_{\omega} \| \dot{\mathfrak{t}}=\check{\mathfrak{t}}$.

Remark 3.7. The fact that $\mathrm{MA}(\sigma$-centered $)$ is preserved under the addition of a single Cohen real is known as Roitman's Theorem [5]. By Bell's Theorem [1], Roitman's Theorem states that $\mathscr{C}_{\omega} \Vdash \dot{\mathfrak{p}} \geq \check{\mathfrak{p}}$.

\section{REFERENCES}

[1] M. Bell, On the combinatorial principle P(c), Fund. Math. 114 (1981), no. 2, 149-157. MR 83e:03077

[2] E. K. van Douwen, The integers and topology, Handbook of set-theoretic topology (K. Kunen and J. E. Vaughan, eds.) North-Holland, Amsterdam-New York, 1984, p. 116. MR 87f:54008

[3] I. Farah and S. Todorčević, Some applications of the method of forcing, Yenisey Publ. Co., Moscow, 1995, p. 12. CMP 98:05

[4] Z. Piotrowski and A. Szymański, Some remarks on category in topological spaces, Proc. Amer. Math. Soc. 101 (1987), no. 1, 156-160. MR 88g:54007

[5] J. Roitman, Correction to: "Adding a random or a Cohen real: topological consequences and the effect on Martin's axiom”, Fund. Math. 129 (1988), no. 2, 141. MR 89f:03045

Department of Mathematics, University of Toronto, Toronto, Canada

E-mail address: hirschor@math.toronto.edu 